\title{
Dynamic Changes of Tyrosine Hydroxylase and Dopamine Concentrations in the Ventral Tegmental Area-Nucleus Accumbens Projection During the Expression of Morphine-Induced Conditioned Place Preference in Rats
}

\author{
Jing Liang $\cdot$ Si-Si Ma $\cdot$ Yi-Jing Li $\cdot$ Xing-Jie Ping $\cdot$ \\ Ling Hu $\cdot$ Cai-Lian Cui
}

Received: 14 January 2012/Revised: 7 February 2012 / Accepted: 20 February 2012/Published online: 7 March 2012

(C) Springer Science+Business Media, LLC 2012

\begin{abstract}
Our previous study demonstrated that morphine dose- and time-dependently elevated dopamine (DA) concentrations in the nucleus accumbens (NAc) during the expression of morphine-induced conditioned place preference (CPP) in rats. However, still unknown are how DA concentrations dynamically change during the morphineinduced CPP test and whether tyrosine hydroxylase (TH) activity in the ventral tegmental area (VTA) plays a vital role in this process. In the present study, we measured dynamic changes in TH and phosphorylated TH serine 40 $\left(\mathrm{pTH} \mathrm{Ser}{ }^{40}\right.$ ) and $\mathrm{pTH} \mathrm{Ser}{ }^{31}$ proteins in the VTA, and DA concentrations in the NAc at $5 \mathrm{~min}$ intervals during a 30 min morphine-induced CPP test. Rats that underwent morphine-induced CPP training significantly preferred the morphine-paired chamber during the CPP expression test, an effect that lasted at least $30 \mathrm{~min}$ in the drug-free state. DA concentrations in the NAc markedly increased at $15 \mathrm{~min}$ when the rats were returned to the CPP boxes to assess the expression of preference for the previously drugpaired chamber. DA concentrations then declined $2 \mathrm{~h}$ after the CPP test. TH and pTH Ser ${ }^{40}$ levels, but not pTH Ser ${ }^{31}$ levels, in the VTA were enhanced during the CPP test. These results indicated that $\mathrm{TH}$ and the phosphorylation of
\end{abstract}

Jing Liang and Si-Si Ma contributed equally to this work.

J. Liang $\cdot$ S.-S. Ma $\cdot$ Y.-J. Li $\cdot$ X.-J. Ping $\cdot$ L. Hu •

C.-L. Cui $(\square)$

Neuroscience Research Institute and Department of

Neurobiology, Peking University Health Science Center,

38 Xueyuan Road, Beijing 100083, People's Republic of China

e-mail: clcui@bjmu.edu.cn; clcuimclean@gmail.com

J. Liang

Key Laboratory of Mental Health, Institute of Psychology,

Chinese Academy of Sciences, Beijing 100101,

People's Republic of China
TH $\mathrm{Ser}^{40}$ in the VTA may be responsible for DA synthesis and release in the NAc during the behavioral expression of conditioned reward elicited by a drug-associated context.

Keywords Morphine Conditioned place preference (CPP) · Dopamine (DA) · Tyrosine hydroxylase (TH)

\section{Introduction}

Morphine, an opioid receptor agonist, triggers the release of dopamine (DA) in the nucleus accumbens (NAc), thus activating the "reward circuit" of the brain and producing the subjective feelings of euphoria during the early stage of administration $[1,2]$. When explicitly pairing the pleasure induced by morphine with a context or set of contextual cues, preference for that context emerges even when the drug is absent.

DA is one of the neurotransmitters that are implicated in drug addiction. The synthesis and release of DA is not simply the neural substrate of the rewarding effects induced by natural rewards and drugs. It is also involved in other crucial elements of associative learning, such as incentive salience, outcome expectation and memory consolidation [3-5]. Despite the widespread expression of DA receptors in the brain, the NAc remains the focus of addiction studies. The NAc receives glutamatergic projections from multiple cortical areas and a dopaminergic projection from the ventral tegmental area (VTA) [6, 7]. Dopaminergic signals can induce the synaptic plasticity of glutamatergic transmission, thus facilitating the formation of associations $[8,9]$. Compared with natural rewards, increases in DA in the NAc induced by drugs have a higher amplitude and faster rise time, which can accelerate synaptic plasticity and result in stronger associations between drugs and drug-related cues 
[10]. After the association is formed, without drugs being present, the drug user can engage in drug-seeking behavior when they are reexposed to the drug-related environment. DA neurons in the NAc show an increase in burst firing upon conditioned cue presentation $[11,12]$.

A previous study from our laboratory revealed that morphine dose- and time-dependently elevated DA concentrations in the NAc during the expression and retention of morphine-induced conditioned place preference (CPP) [13]. Tyrosine hydroxylase $(\mathrm{TH})$, a rate-limiting enzyme in DA synthesis, is mainly located in the soma of DA neurons (i.e., VTA) [14, 15]. Studies have shown that TH activity can be accelerated within a short time, and several trans-acting factors (e.g., Fos, Jun and cyclic adenosine monophosphate response element binding protein) bind to $c i s$ elements within the promoter region of the TH gene and affect its transcription [16, 17]. Cleavage of the proenzyme from the inactive form to the functional form might also occur. Phosphorylated TH serine $40\left(\mathrm{pTH} \mathrm{Ser}^{40}\right)$ and $\mathrm{pTH} \mathrm{Ser}{ }^{31}$ are two major active forms of TH, the levels of which are positively related to the speed of the reaction [18]. New questions have been asked about how DA levels change during the expression of morphine-induced CPP, and whether TH activity in the VTA plays a vital role in this process. The aim of the present study was to determine the dynamic changes in DA concentrations that occur in the NAc, and TH activation in the VTA (phosphorylation levels of $\mathrm{TH}$ at the serine 31 and 40 sites), during the morphine-induced CPP test.

\section{Materials and Methods}

Subjects

All of the experiments were performed using male Sprague-Dawley rats obtained from the Experimental Animal
Center, Peking University, weighing $230-250 \mathrm{~g}$ at the beginning of the study. The animals were housed four per cage under a $12 \mathrm{~h} / 12 \mathrm{~h}$ light/dark cycle (lights on at 7:00 $\mathrm{am}$, lights off at 7:00 pm) with food and water available. The room temperature was maintained at $24 \pm 1{ }^{\circ} \mathrm{C}$ with $45-55 \%$ relative humidity. All of the experimental procedures were approved by the Animal Use Committee of Peking University Health Science Center.

The rats were divided into three groups (Table 1): (1) Naive group (rats were given $2 \mathrm{ml} / \mathrm{kg}$ saline at 7:00 am and 7:00 pm every day for 4 days), (2) Morphine injection only group (rats were given $2 \mathrm{ml} / \mathrm{kg}[2 \mathrm{mg} / \mathrm{ml}]$ morphine at 7:00 am and7:00 pm every day for 4 days in parallel with the Morphine CPP group), and (3) Morphine CPP groups. For a detailed description of the CPP training schedule, see below. The Morphine CPP groups were further divided into seven subgroups according to different testing time-points: $0,5,10,15,20,25$, and 30 min groups.

\section{Apparatus}

Conditioning was conducted in black rectangular polyvinyl chloride boxes $\left(795 \times 230 \times 250 \mathrm{~mm}^{3}\right)$ that contained three chambers separated by guillotine doors. Two large black conditioning chambers (Chambers A and C, $280 \times$ $220 \times 225 \mathrm{~mm}^{3}$ ) were separated by a small gray center choice chamber (Chamber B, $135 \times 220 \times 225 \mathrm{~mm}^{3}$ ). On the wall of Chamber A were four light-emitting diodes (LEDs) that formed a square. On the wall of Chamber C were four LEDs that formed a triangle. Chamber A had a stainless steel mesh floor $\left(225 \times 225 \mathrm{~mm}^{2}\right)$. Chamber C had a stainless-steel rod floor, with the rods spaced $15 \mathrm{~mm}$ apart. Chamber B had a plain floor. Fourteen photobeams were placed across the chambers, spaced $47.5 \mathrm{~mm}$ apart.

Table 1 Experimental design

\begin{tabular}{lclll}
\hline Group & Samples & Treatment & Duration of CPP test & Harvest \\
\hline Naive & 3 & No treatment & N/A & $\begin{array}{c}\text { Harvest after all the CPP-duration 0 group } \\
\text { were harvested }\end{array}$ \\
Morphine injection only & 3 & $\begin{array}{c}\text { Treated with morphine or saline only } \\
\text { in the home cage }\end{array}$ & N/A \\
$\begin{array}{l}\text { CPP 0 min duration } \\
\text { (without CPP test) }\end{array}$ & 10 & Morphine-induced CPP conditioning & 0 min & Harvest 24 h after last CPP training \\
CPP 5 min duration & 10 & Morphine-induced CPP conditioning & 5 min & Harvest immediately after CPP test \\
CPP 10 min duration & 10 & Morphine-induced CPP conditioning & 10 min & \\
CPP 15 min duration & 28 & Morphine-induced CPP conditioning & 15 min & \\
CPP 20 min duration & 10 & Morphine-induced CPP conditioning & 20 min & \\
CPP 25 min duration & 10 & Morphine-induced CPP conditioning & 25 min & \\
CPP 30 min duration & 10 & Morphine-induced CPP conditioning & 30 min & \\
\hline
\end{tabular}

$C P P$ conditioned place preference, N/A not applicable 
Using a computer interface, the time spent in each chamber was recorded by infrared beam crossings.

\section{Conditioned Place Preference Procedures}

The CPP experiments were conducted using procedures described previously [19], with the exception of minor modifications described below.

\section{Preconditioning Test}

The rats were handled for at least 3 days before the experiments. On day 0 , the rats were placed in Chamber B with the guillotine doors removed, which allowed them to access the entire apparatus for $15 \mathrm{~min}$, and the time spent in each side was recorded. The CPP score was calculated as the following: Time Spent in Chamber $\mathrm{A}(\mathrm{C}) /($ Time Spent in Chamber $\mathrm{A}+$ Time Spent in Chamber $\mathrm{C}$ ). The rats with a CPP score less than 0.45 or greater than 0.55 were considered naturally biased and were excluded from the subsequent experiments.

\section{Conditioning}

Beginning on day 1, the animals were scheduled for a 45 min training period twice per day (9:00 am and 3:00 $\mathrm{pm}$ ) for 4 days. The animals received $2 \mathrm{mg} / \mathrm{kg}$ morphine (i.p.) before being confined to one side (e.g., Chamber A) and a saline injection (vehicle) before being confined to the other side (e.g., Chamber C). The drug-paired sides were counter-balanced among all groups.

\section{Post-Conditioning Test Phase}

On day 5, all of the animals were placed in the choice chamber with the guillotine doors removed to allow access to the entire apparatus for $15 \mathrm{~min}$, and the time spent in each side was recorded. The CPP score was calculated as the following: Time Spent in Morphine-Paired Chamber/(Time Spent in MorphinePaired Chamber + Time Spent in Saline-Paired Chamber).

\section{Tissue Dissection and Preparation}

The rats were decapitated at the times shown in Table 1. The brains were removed and placed on an ice-cooled plate for dissection of the NAc and VTA according to a stereotaxic atlas [20]. The tissue samples were frozen in liquid nitrogen and stored at $-80^{\circ} \mathrm{C}$ until analysis.

High-Performance Liquid Chromatography for DA Determination

The tissue samples for high-performance liquid chromatography (HPLC) were weighed and placed in $0.5 \mathrm{ml}$ plastic tubes that contained ice-cooled perchloric acid $(200 \mu \mathrm{l}, 0.4 \mathrm{M})$, homogenized for $10 \mathrm{~s}$ using ultrasound $(0.5 \mathrm{~Hz})$ and centrifuged for $20 \mathrm{~min}$ at $15,000 \times g$ at $4^{\circ} \mathrm{C}$. The supernatant was passed through a $0.2 \mu \mathrm{m}$ filter and kept at $4^{\circ} \mathrm{C}$ until the HPLC analysis.

DA content in NAc tissue was analyzed using reversedphase ion-pair chromatography combined with electrochemical detection under isocratic conditions [21]. The sixchannel detector potentials were set at $+50,100,200,300$, 400 , and $500 \mathrm{mV}$ using a glassy carbon electrode and an $\mathrm{Ag} / \mathrm{AgCl}$ reference electrode. The mobile phase $(0.6 \mathrm{mM}$ 1-octanesulfonic acid, $0.27 \mathrm{mM} \mathrm{Na}_{2}$ EDTA, $0.043 \mathrm{M}$ triethylamine and $50 \mathrm{ml}$ acetonitrile/L, adjusted to $\mathrm{pH} 2.95$ with $\mathrm{H}_{3} \mathrm{PO}_{4}$ ) was delivered at a flow rate of $0.5 \mathrm{ml} / \mathrm{min}$ onto the reverse-phase column $(125 \mathrm{~mm} \times 3 \mathrm{~mm}$ with $5 \mathrm{~mm} \times 3 \mathrm{~mm}$ pre-column filled with Nucleosil 120-3 $\mathrm{C}_{18}$; Knauer, Berlin, Germany). Ten microliter aliquots were injected using an autoinjector with the cooling module set at $4^{\circ} \mathrm{C}$. The data were calculated against an external standard calibration.

Western Blotting for Tyrosine Hydroxylase, pTH Ser ${ }^{31}$, and $\mathrm{pTH} \mathrm{Ser}^{40}$

Tissue samples for Western blotting were homogenized in 10 volumes of RIPA (Applygene, Beijing, China). The homogenate was then centrifuged at 12,000 rotations per minute for $10 \mathrm{~min}$. The resulting supernatant was used as the total protein. Protein concentrations were determined using the BCA assay (Pierce, Rockford, IL, USA).

Equivalent amounts of membrane preparations $(60 \mu \mathrm{g})$ for each sample were resolved by $10 \%$ sodium dodecyl sulfate polyacrylamide gel electrophoresis (SDS-PAGE). After electrophoresis, the proteins were transferred to polyvinylidene fluoride (PVDF) membranes. The membranes were incubated in Tris-buffered saline (TBS) with $0.1 \%$ Tween-20 (TBST) that contained 5\% nonfat milk for $1 \mathrm{~h}$ at room temperature with agitation to block nonspecific binding. The membrane was incubated with primary antibody diluted in TBS (TH [1:4000, Sigma], pTH Ser ${ }^{31}$ and pTH Ser ${ }^{40}$ [1:500, Abcam]) that contained 5\% nonfat dried milk overnight at $4^{\circ} \mathrm{C}$. The membrane was then washed three times for $5 \mathrm{~min}$ in TBST followed by $1 \mathrm{~h}$ incubation at room temperature with horseradish peroxidase-conjugated rabbit anti-goat immunoglobulin G (IgG; Zhongshan Biotechnology, Beijing, China) diluted 1:10,000 in TBS that contained $5 \%$ nonfat dried milk. After this incubation, the membranes were washed three times for $5 \mathrm{~min}$ in TBST. The antigen-antibody peroxidase complex was then detected by enhanced chemiluminescence (Pierce) according to the manufacturer's instructions and visualized by exposure to Kodak film (Eastman Kodak, Kodak, NJ, USA). The bands on the autoradiogram were quantified 
with the TotalLab 2.01 Analysis System (Phoretix, UK), and the optical density of the corresponding $\beta$-actin band corrected the optical density of each target band. The values were expressed as a percentage of control.

\section{Drugs}

Morphine hydrochloride was purchased from the First Pharmaceutical Factory, Shenyang, China. Morphine hydrochloride was dissolved in saline.

\section{Data Analysis}

CPP scores represent an index of place preference for each rat, calculated by dividing the time spent in the drug-paired compartment by the time spent in both conditioning compartments. The data were analyzed using Prism 4.0 software (GraphPad, San Diego, CA, USA). The results were expressed as mean \pm SEM (see below for details). The data were analyzed with a two-tailed $t$ test, one-way analysis of variance (ANOVA) followed by the NewmanKeuls post hoc test, and two-way ANOVA or repeatedmeasures ANOVA followed by the Bonferroni post hoc test. The accepted level of statistical significance was $p<0.05$.

\section{Results}

Morphine-Paired Environment Induced Preference Behaviors and Changes in DA Concentrations in the Nucleus Accumbens

After 4 days of CPP training, the rats that underwent conditioning were divided into seven groups and subjected to post-conditioning preference tests in a drug-free state for $0,5,10,15,20,25$ and $30 \mathrm{~min}$. This schedule was designed to detect dynamic changes in behavioral preference and DA concentration in the NAc during reexposure to the drug-related context. On the post-test day, the rats were reintroduced to the conditioning chamber with free access to both the morphine- and saline-paired sides. Figure 1 shows morphine-induced CPP scores during the $30 \mathrm{~min}$ test. The repeated-measures ANOVA revealed no significant effect of Treatment $\left(F_{5,54}=0.9477, p=0.458\right)$ but a significant effect of Time $\left(F_{1,54}=72.11, p<0.0001\right)$ and a trend toward a significant Treatment $\times$ Time interaction $\left(F_{5,54}=2.14, p=0.075\right)$. The Bonferroni post hoc test showed that rats tested for $>15$ min exhibited a significant preference for the morphine-paired context, reflected by spending more time in that chamber. The rats tested for 5 and 10 min spent nearly the same time in the morphineand saline-paired contexts. General arousal and novelty seeking might account for the lack of preference during the first 5-10 min of the test. The apparent preference after 15 min suggested that the association between morphineinduced euphoria and contextual cues was established.

Subsequently, HPLC was performed to evaluate DA levels in the NAc. Figure 2a shows the changes in DA content in the NAc when the rats were placed again in the CPP apparatus for $30 \mathrm{~min}$ in $5 \mathrm{~min}$ blocks. The one-way ANOVA followed by the Newman-Keuls post hoc test revealed a significant effect of Treatment $\left(F_{6,45}=2.795\right.$, $p=0.0237$ ), and DA content in the NAc in rats tested for 15 min was markedly higher than in the control group without re-exposure to the CPP apparatus after morphine conditioning $(p<0.05)$. The results suggested that peak DA levels in the NAc occurred when preference began to emerge. The enhanced DA concentration decreased to the level of the control group $2 \mathrm{~h}$ after the CPP test (Fig. 2b; $p<0.05$, one-way ANOVA followed by Newman-Keuls post hoc test; $F_{4,37}=3.946, p<0.0001$ ).

Dynamic Changes in TH and TH Phosphorylation Levels in the VTA During the CPP Test

To determine whether increased DA concentration in the NAc resulted from elevated DA synthesis in the VTA where the soma of DA neurons reside, VTA tissues were collected and processed. TH, pTH Ser ${ }^{40}$, and pTH $\mathrm{Ser}^{31}$ levels were analyzed using Western blotting. As shown in Fig. 3a, TH levels in the VTA showed an inverted U-shaped curve with increased post-test time $\left(F_{6}, 20=\right.$ $6.016, p=0.0027)$. Peak TH levels in the VTA overlapped with peak DA content in the NAc, but remained at a high level from the 15 to $25 \mathrm{~min}$ time points $(p<0.05)$. After 15 min of the CPP test, we found that the amount of TH in

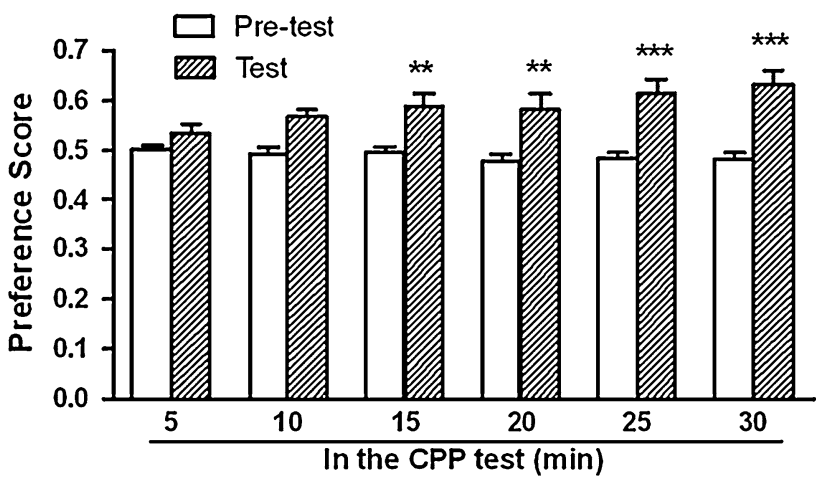

Fig. 1 Morphine-induced conditioned place preference score during the 30 min test $(n=10)$. After 4 days of CPP conditioning, the rats were divided into seven groups and tested for 5,10,15,20, 25, and $30 \mathrm{~min}$. Preference scores were expressed as mean \pm SEM. The data were analyzed using two-way repeated-measures ANOVA followed by the Bonferroni post hoc test. $* * p<0.01, * * * p<0.001$, compared with corresponding pretest score. $C P P$ conditioned place preference 
(a)

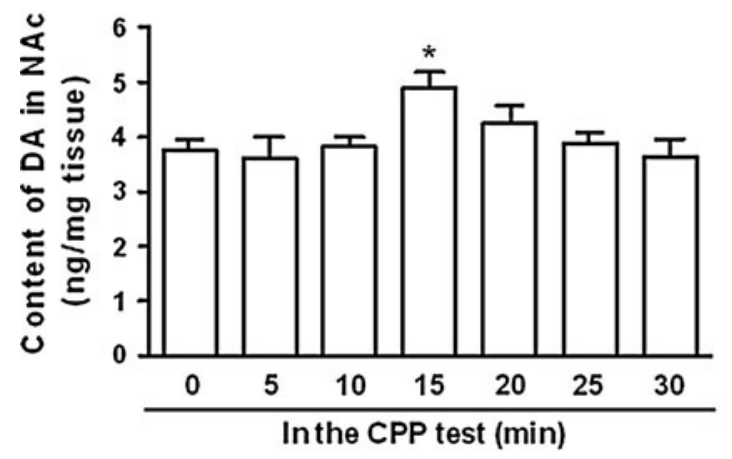

Fig. 2 Fluctuation of DA concentration in the NAc during the 30 min CPP test (a, $n=5-9)$ and change $3 \mathrm{~h}$ after the 15 min CPP test $(\mathbf{b}, n=6-9)$. The data were analyzed using one-way ANOVA followed by the Newman-Keuls post hoc test. $* p<0.05$, **p $p<0.01$, (b)

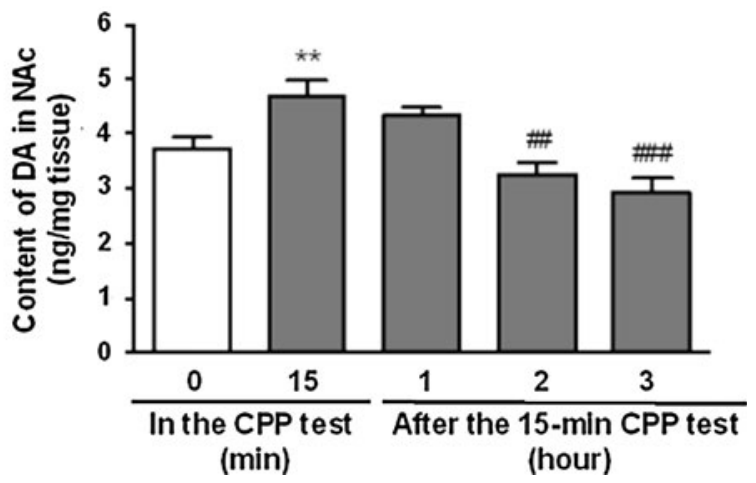

compared with 0 min group, which received only CPP conditioning without a post-test; ${ }^{\# \#} p<0.01,{ }^{\# \# \#} p<0.001$, compared with $15 \mathrm{~min}$ group. $D A$ dopamine, $N A c$ nucleus accumbens
Fig. 3 Dynamic change in $\mathrm{TH}$ content in the VTA during the 30 min test (a, $n=3), 3 \mathrm{~h}$ after the 15 min CPP test $(\mathbf{b}, n=3)$, and elicited by a morphine injection (c, $n=3)$. The data were analyzed using one-way ANOVA followed by the Newman-Keuls post hoc test. $* p<0.05, * * p<0.01$, compared with 0 min group. Representative results of Western blotting are shown on the bottom of the panel. $\mathrm{TH}$ tyrosine hydroxylase (a)
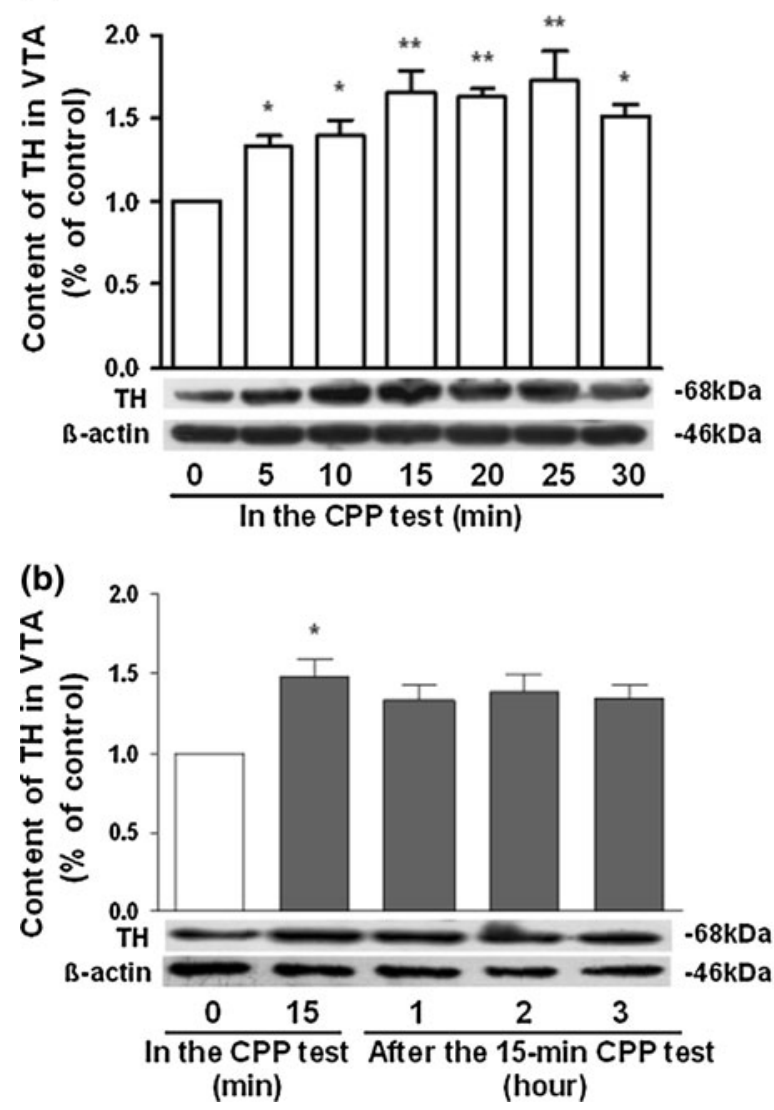

(c)

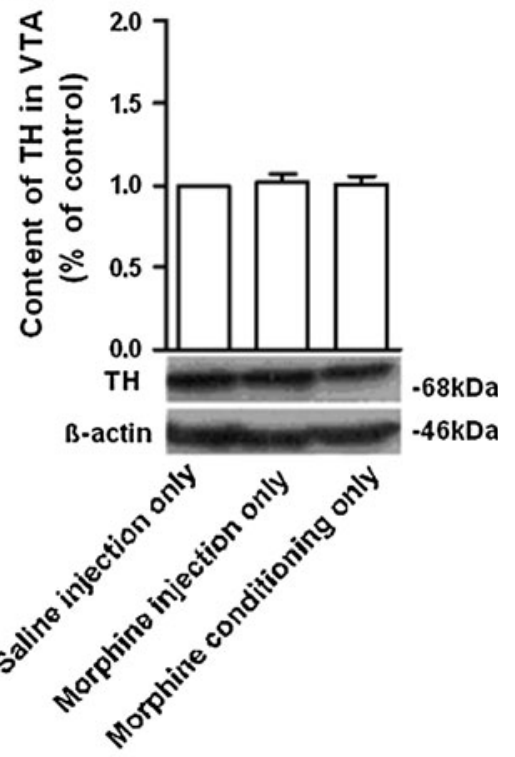

the VTA remained at a high level for at least $3 \mathrm{~h}$, although the difference was not significant (Fig. 3b). Neither morphine injections in the home cage nor morphine conditioning training without the CPP test altered TH levels in the VTA (Fig. 3c), with no significant differences between any of the groups (one-way ANOVA, $p=0.9452$ ).
Figure 4a shows the changes in pTH Ser ${ }^{40}$ levels in the VTA during the $30 \mathrm{~min}$ CPP test. One-way ANOVA of the data in the 0-30 min groups revealed a significant effect of Treatment $\left(F_{6,22}=12.41, p<0.0001\right)$. The Newman-Keuls post hoc test showed that $\mathrm{pTH} \mathrm{Ser}{ }^{40}$ levels increased when the animals were placed in the conditioning chamber for $5 \min (p<0.05$, compared with the $0 \mathrm{~min}$ group) and decreased $25 \mathrm{~min}$ later. 
Figure $4 \mathrm{~b}$ illustrates the changes in $\mathrm{pTH} \mathrm{Ser}{ }^{31}$ levels in the VTA when the rats stayed in the test apparatus for $30 \mathrm{~min}$. Oneway ANOVA of the data in the 0-30 min groups revealed a significant effect of Treatment $\left(F_{6,25}=2.845, p=0.0377\right)$. This significant difference was only observed between the 15 min and 25 min groups ( $p<0.05$, Newman-Keuls post hoc test; Table 2).

\section{Discussion}

Dynamic Changes in DA Concentrations in the NAc during the Expression of Morphine-Induced CPP

An early view of DA function was that it signaled the experience of addictive drugs as a hedonic neurotransmitter, but this viewpoint has been challenged by pharmacological

\section{(a) Level of $\mathrm{pTH}$-ser ${ }^{40}$ in VTA}

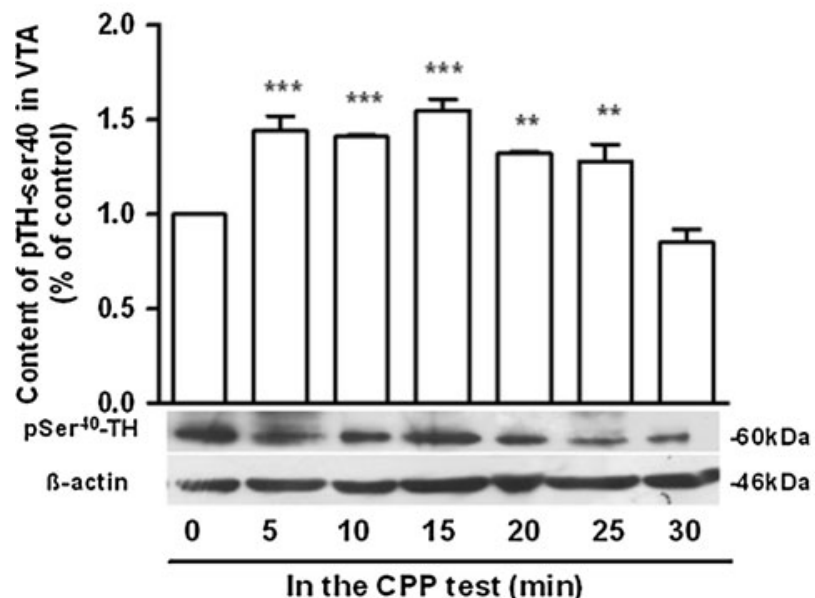

Fig. 4 Change in phosphorylated TH at $\operatorname{Ser}^{40}(\mathbf{a}, n=3-4)$ and phosphorylated TH at $\operatorname{Ser}^{31}(\mathbf{b}, n=3)$ in the VTA during the $30 \mathrm{~min}$ test. The data were analyzed using one-way ANOVA followed by the Newman-Keuls post hoc test. $* * p<0.01$, ***p $p<0.001$, compared blockade and genetic and lesion studies, in which animals maintained their preference for reward, such as morphine, even after DA depletion [22-24]. Most evidence from electrophysiological and microdialysis studies now suggest that, rather than mediating the unconditioned effects of reward, DA is involved in the anticipation of reward or behavioral activation $[25,26]$. These results, together with our previous findings [13], suggest that DA synthesis may participate in the reward-associated conditioned response. Thus, in the present study, based on the preference of rats for the morphine-paired environment for $30 \mathrm{~min}$, we assessed DA content in the NAc in 5 min blocks during the CPP test. Peak DA content was observed at the 15 min time point of the test, and then rapidly decreased and remained at baseline levels during the subsequent 15 min test. However, if the rats were removed from the test chamber after $15 \mathrm{~min}$, then this high DA content could be maintained for $1 \mathrm{~h}$. These

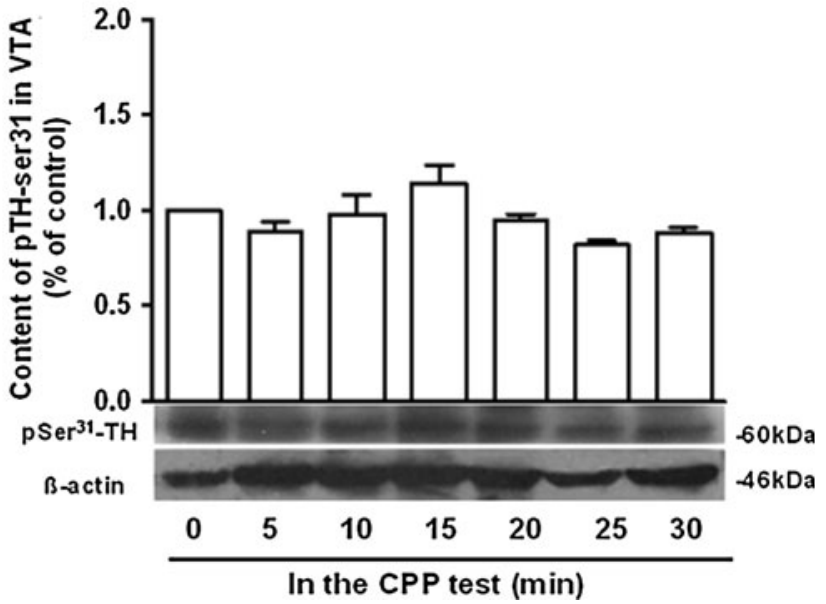

with 0 min group. Representative results of Western blotting are shown on the bottom of the panel. pTH-ser ${ }^{40}$, phosphorylated TH at serine 40 site; $\mathrm{pTH}-\mathrm{ser}^{31}$, phosphorylated $\mathrm{TH}$ at serine 31 site

Table 2 Summary of significant results

\begin{tabular}{|c|c|c|c|c|c|c|c|c|c|}
\hline \multirow[t]{2}{*}{ Measurement } & \multicolumn{6}{|c|}{ Duration of CPP test } & \multicolumn{3}{|c|}{ After 15 min CPP test } \\
\hline & $5 \mathrm{~min}$ & $10 \mathrm{~min}$ & $15 \mathrm{~min}$ & $20 \mathrm{~min}$ & $25 \mathrm{~min}$ & $30 \mathrm{~min}$ & $1 \mathrm{~h}$ & $2 \mathrm{~h}$ & $3 \mathrm{~h}$ \\
\hline Morphine-induced CPP & & & $* *$ & $* *$ & $* * *$ & $* * *$ & & & \\
\hline NAc DA content & & & $*$ & & & & & $\downarrow$ & $\downarrow$ \\
\hline VTA TH level & $*$ & $*$ & $* *$ & $* *$ & $* *$ & $*$ & & & \\
\hline $\begin{array}{l}\text { VTA pTH Ser }{ }^{40} \text { level } \\
\text { VTA pTH } \text { Ser }^{31} \text { level }\end{array}$ & $* * *$ & $* * *$ & $* * *$ & $* *$ & $* *$ & & & & \\
\hline
\end{tabular}

$V T A$ ventral tegmental area, $N A c$ nucleus accumbens, $T H$ tyrosine hydroxylase

$* p<0.05$

$* * p<0.01$

$* * * p<0.001$ 
findings, to some extent, are consistent with the results of previous studies that investigated natural reward. When animals are reexposed to natural reward-related cues, DA neurons are excited in the VTA [11, 27, 28], subsequently leading to DA release in the NAc [12, 29] without the presence of the reward. However, if the animal cannot obtain the rewarding drug after exposure to reward-related stimuli, then the electrophysiological response of dopaminergic neurons decreases to a level that is even lower than physiological levels [30]. Our results predicted that when the rats that underwent morphine conditioning were placed in the CPP apparatus for a $15 \mathrm{~min}$ post-conditioning test, their "craving" for the drug experience elicited by morphinerelated cues reached an apex, driving DA synthesis and increasing DA release in the NAc. After fully learning the predictive salience of conditioned stimuli, the DA signal is enhanced when the animal is reexposed to the conditioned stimulus (e.g., drug-related environmental cues in the present study). The findings from microdialysis of DA in CPP model strengthen this implication. Exposure to cues previously paired with drug administration produced a significant increase in extracellular accumbens DA levels during 5-25 min with a peak at $10 \mathrm{~min}$ [31]. Twenty-minute drugpaired environment elicited a robust increase of DA release in NAc and the change positively correlated with preference degree [32]. However, due to showing an integrative change including release, synthesis and transmission of DA, tissue DA content can not meet a synchronous change with extracellular DA and TH level.

Upregulation of $\mathrm{TH}$ and $\mathrm{pTH} \mathrm{Ser}^{40}$ Levels Contribute to DA Synthesis in the Expression of MorphineInduced CPP

In the present study, we found that TH content in the VTA first increased and then decreased, with a high plateau between the 15 and 25 min time points during the CPP test. This high level of $\mathrm{TH}$ protein was maintained for at least $3 \mathrm{~h}$ after the $15 \mathrm{~min}$ CPP test. These results indicated that reexposure to morphine-related environmental cues may induce the synthesis or modification of TH. The increase in TH in the VTA may also contribute to the increase in DA concentration in the NAc. Recent studies demonstrated that TH gene-deficient animals exhibited deficits in performing operant conditioning tasks $[33,34]$, suggesting that $\mathrm{TH}$ is required in conditioned learning and memory processes. Additionally, the present study showed that in the groups that received a morphine injection only, or morphine conditioning without the test, TH levels in the VTA did not significantly change compared with the saline group. This is consistent with a previous report [35].

Alterations in DA content in the NAc may not depend on changes in total TH levels solely in the VTA. The activation of $\mathrm{TH}$ is a more important factor for DA synthesis. Phosphorylated TH proteins are the active forms of TH. To date, four TH phosphorylation sites have been found: $\operatorname{Ser}^{8}, \operatorname{Ser}^{19}, \operatorname{Ser}^{31}$, and $\operatorname{Ser}^{40}$ [30]. Among them, $\mathrm{Ser}^{31}$ and $\mathrm{Ser}^{40}$ are believed to be directly involved in DA synthesis because increases in the phosphorylation of $\mathrm{TH}$ at these two sites enhance the catalytic activity of $\mathrm{TH}$ [36, 37]. Normally, the phosphorylation of $\mathrm{Ser}^{40}$ and $\mathrm{Ser}^{31}$ occurs at low levels, and DA synthesis does not occur until the phosphorylation of $\mathrm{TH}$ at the $\mathrm{Ser}^{40}$ and $\mathrm{Ser}^{31}$ sites occurs [38].

In the present study, the $\mathrm{Ser}^{40}$ phosphorylation of $\mathrm{TH}$ in the VTA increased as soon as the rats were reexposed to the morphine-paired environment and began to decrease after $15 \mathrm{~min}$ in the CPP test. The pattern of $\mathrm{Ser}^{40}$ phosphorylation is similar to the pattern of total TH levels. Both occur in an inverted U-shaped pattern. Nevertheless, the phosphorylation of $\mathrm{TH}$ changes faster than the expression of TH protein. These results may be explained by the fact that protein modification is faster than protein synthesis. During the first $15 \mathrm{~min}$ of the post-conditioning test, the increased $\mathrm{Ser}^{40}$ phosphorylation of $\mathrm{TH}$ in the VTA might be caused by the following: (1) increased TH content in the VTA (however, as mentioned above, TH phosphorylation levels are normally low, so this possibility may be discarded), (2) reexposure to morphine-related environmental cues induces $\mathrm{Ser}^{40}$ phosphorylation. During the second 15 min period of the post-conditioning test, the continuous decrease in $\operatorname{Ser}^{40}$ phosphorylation may reflect the fact that the animals were exposed to conditioned stimuli (i.e., a morphine-related environment) but did not obtain the drug reward (i.e., the euphoria induced by morphine) as expected. Several research groups reported that, under this condition, DA release in the NAc deceases because of the depression of DA neurons in the VTA [11]. TH phosphorylation is also downregulated by some negative feedback mechanisms at this time.

The $\mathrm{Ser}^{31}$ phosphorylation of TH in the VTA did not significantly change during the post-conditioning test. Distinct upstream signal transduction pathways may contribute to the differential response pattern of $\mathrm{Ser}^{31}$ and $\mathrm{Ser}^{40}$ phosphorylation during this process [30]. Our findings indicate that the phosphorylation of $\mathrm{TH}$ at the Ser ${ }^{40}$ site is more closely related than the phosphorylation of $\mathrm{TH}$ at the $\mathrm{Ser}^{31}$ site to the behaviors induced by reexposure and DA release [39].

In conclusion, the results of the present study revealed the dynamic changes in $\mathrm{TH}$ and DA concentrations that occur in the VTA-NAc projection during the expression of morphine-induced CPP in rats. $\mathrm{Ser}^{40}$ phosphorylation, but not $\mathrm{Ser}^{31}$ phosphorylation, of $\mathrm{TH}$ in the VTA exhibited similar changes that preceded the changes in $\mathrm{TH}$ content. The phosphorylation of $\mathrm{TH}$ (the key enzyme of DA 
synthesis), especially at the $\mathrm{Ser}^{40}$ site, may be responsible for changes in DA content in the NAc during the expression of conditioning behaviors associated with reward.

Acknowledgments This work was supported by grants from the National Natural Science Foundation (30970933) and National Basic Research Program (2009CB522003).

\section{References}

1. Wise RA (1989) Opiate reward: sites and substrates. Neurosci Biobehav Rev 13:129-133

2. Hayman SE, Malenka RC, Nestler EJ (2006) Neural mechanisms of addiction: the role of reward-related learning and memory. Annu Rev Neurosci 29:565-598

3. Spanagel R, Weiss F (1999) The dopamine hypothesis of reward: past and current status. Trends Neurosci 22:521-527

4. Pierce RC, Kumaresan V (2006) The mesolimbic dopamine system: the final common pathway for the reinforcing effect of drugs of abuse? Neurosci. Biobehav Rev 30:215-238

5. Dalley JW, Everitt BJ (2009) Dopamine receptors in the learning, memory and drug reward circuitry. Semin Cell Dev Biol 20:403-410

6. Der-Avakian A, Bland ST, Schmid MJ et al (2006) The role of glucocorticoids in the uncontrollable stress-induced potentiation of nucleus accumbens shell dopamine and conditioned place preference responses to morphine. Psychoneuroendocrinology 31:653-663

7. Fenu S, Spina L, Rivas E et al (2006) Morphine-conditioned single-trial place preference: role of nucleus accumbens shell dopamine receptors in acquisition, but not expression. Psychopharmacology 187:143-153

8. Wolf ME, Mangiavacchi S, Sun X (2003) Mechanisms by which dopamine receptors may influence synaptic plasticity. Ann N Y Acad Sci 1003:241-249

9. Kalivas PW, O’Brien C (2008) Drug addiction as a pathology of staged neuroplasticity. Neuropsychopharmacology 33:166-180

10. Opris I, Hampson RE, Deadwyler SA (2009) The encoding of cocaine vs. natural rewards in the striatum of nonhuman primates: categories with different activations. Neuroscience 163:40-54

11. Schultz W (1998) Predictive reward signal of dopamine neurons. J Neurophysiol 80:1-27

12. Phillips AG (2003) Mesocorticolimbic dopamine: a neurochemical link between motivation and memory. Int Congr Ser 1250: 509-526

13. Ma YY, Meng L, Guo CY et al (2009) Dose- and time-dependent, context-induced elevation of dopamine and its metabolites in the nucleus accumbens of morphine-induced CPP rats. Behav Brain Res 204:192-199

14. Bjorklund A, Dunnett SB (2007) Dopamine neuron systems in the brain: an update. Trends Neurosci 30:194-202

15. Iversen SD, Iversen LL (2007) Dopamine: 50 years in perspective. Trends Neurosci 30:188-193

16. Kumer SC, Vrana KE (1996) Intricate regulation of tyrosine hydroxylase activity and gene expression. J Neurochem 67: 443-462

17. Sabban EL, Kvetnansky R (2001) Stress-triggered activation of gene expression in catecholaminergic systems: dynamics of transcriptional events. Trends Neurosci 24:91-98

18. Haycock JW (1990) Phosphorylation of tyrosine hydroxylase in situ at serine 8, 19, 31, and 40. J Biol Chem 265:11682-11691
19. Liang J, Li Y, Ping X, Yu P et al (2006) The possible involvement of endogenous ligands for mu-, delta- and kappa-opioid receptors in modulating morphine-induced CPP expression in rats. Peptides 27:3307-3314

20. Paxinos G, Watson C (1997) The Rat Brain in Stereotaxic Coordinates. Academic Press, London

21. Teismann P, Ferger B (2001) Inhibition of the cyclooxygenase isoenzymes COX-1 and COX-2 provide neuroprotection in the MPTP-mouse model of Parkinson's disease. Synapse 39:167-174

22. Berridge KC, Robinson TE (1998) What is the role of dopamine in reward: hedonic impact, reward learning, or incentive salience? Brain Res Rev 28:309-369

23. Cannon CM, Palmiter RD (2003) Reward without dopamine. J Neurosci 23:10827-10831

24. Hnasko TS, Sotak BN, Palmiter RD (2005) Morphine reward in dopamine-deficient mice. Nature 438:854-857

25. Sunsay C, Rebec GV (2008) Real-time dopamine efflux in the nucleus accumbens core during Pavlovian conditioning. Behav Neurosci 122:358-367

26. Owesson-White CA, Ariansen J, Stuber GD et al (2009) Neural encoding of cocaine-seeking behavior is coincident with phasic dopamine release in the accumbens core and shell. Eur J Neurosci 30:1117-1127

27. Schultz W (2002) Getting formal with dopamine and reward. Neuron 36:241-263

28. Schultz W, Romo R (1990) Dopamine neurons of the monkey midbrain: contingencies of responses to stimuli eliciting immediate behavioral reactions. J Neurophysiol 63:607-624

29. Day JJ, Roitman MF, Wightman RM et al (2007) Associative learning mediates dynamic shifts in dopamine signaling in the nucleus accumbens. Nat Neurosci 10:1020-1028

30. Schultz W (2007) Behavioral dopamine signals. Trends Neurosci 30:203-210

31. Geraslimov MR, Schiffer WK, Gardner EL et al (2001) GABAergic blockade of cocaine-associated cue-induced increases in nucleus accumbens dopamine. Eur J Pharmacol 414:205-209

32. Schiffer WK, Liebling CN, Reiszel C et al (2009) Cue-induced dopamine release predicts cocaine preference: positron emission tomography studies in freely moving rodents. J Neurosci 29: $6176-6185$

33. Denenberg VH, Kim DS, Palmiter RD (2004) The role of dopamine in learning, memory, and performance of a water escape task. Behav Brain Res 148:73-78

34. Robinson S, Rainwater AJ, Hnasko TS et al (2007) Viral restoration of dopamine signaling to the dorsal striatum restores instrumental conditioning to dopamine-deficient mice. Psychopharmacology 191:567-578

35. Beitner-Johnson D, Nestler EJ (1991) Morphine and cocaine exert common chronic actions on tyrosine hydroxylase in dopaminergic brain reward regions. J Neurochem 57:344-347

36. Dunkley PR, Bobrovskaya L, Graham ME et al (2004) Tyrosine hydroxylase phosphorylation: regulation and consequences. J Neurochem 91:1025-1043

37. Colby KA, Thompson TL, Patrick RL (1989) Tyrosine hydroxylase phosphorylation in rat brain striatal synaptosomes. Brain Res 478:103-111

38. Salvatore MF, Garcia-Espana A, Goldstein M et al (2000) Stoichiometry of tyrosine hydroxylase phosphorylation in the nigrostriatal and mesolimbic systems in vivo: effects of acute haloperidol and related compounds. J Neurochem 75:225-232

39. Shin SS, Bray ER, Zhang CQ et al (2011) Traumatic brain injury reduces striatal tyrosine hydroxylase activity and postassiumevoked dopamine release in rats. Brain Res 1369:208-215 